\title{
On the shape derivative for problems of Bernoulli type
}

\author{
J. HASLINGER \\ Department of Numerical Analysis, Charles University, \\ Sokolovská 83, 18675 Praha 8, Czech Republic \\ E-mail: hasling@karlin.mff.cuni.cz \\ K. ITO \\ Department of Mathematics, North Carolina State University, \\ Raleigh, NC 27695, USA \\ E-mail: kito@unity.ncsu.edu \\ T. KozUBEK \\ Department of Applied Mathematics, VSB-Technical University Ostrava, \\ 17. listopadu 15, 70833 Ostrava 8, Czech Republic \\ E-mail: tomas.kozubek@vsb.cz \\ K. KUnISCH AND G. PEICHL \\ Department for Mathematics and Scientific Computing, University of Graz, \\ Heinrichstrasse 36, A-8010 Graz, Austria \\ E-mail:karl.kunisch@uni-graz.at, gunther.peichl@uni-graz.at
}

[Received 20 February 2008 and in revised form 27 November 2008]

The shape derivative of the cost functional in a Bernoulli-type problem is characterized. The calculation of the derivative of the cost does not use the shape derivative of the state variable and is achieved under mild regularity conditions on the boundary of the domain.

\section{Introduction}

In this note we carry out a sensitivity analysis with respect to the boundary $\Gamma$ in the following shape optimization problem:

$$
\min _{\Gamma} J(\Gamma) \equiv \frac{1}{2} \int_{\Gamma}\left(\frac{\partial u}{\partial n}-Q\right)^{2} \mathrm{~d} \sigma,
$$

where $u$ is the solution of the Dirichlet problem

$$
\begin{aligned}
& -\Delta u=0 \quad \text { on } \Omega \text {, } \\
& u=g \quad \text { on } \Gamma_{d} \text {, } \\
& u=0 \quad \text { on } \Gamma \text {. }
\end{aligned}
$$

Above, $\Omega \subset \mathbb{R}^{2}$ denotes a doubly connected, bounded domain of class $C^{1,1}$ the boundary of which consists of a fixed component $\Gamma_{d}$ and another component $\Gamma$ such that $\operatorname{dist}\left(\Gamma_{d}, \Gamma\right)>0, Q \in \mathbb{R}$ and $g \in H^{3 / 2}\left(\Gamma_{d}\right)$. 
Problems of this type arise for example in the numerical realization of Bernoulli free boundary value problems (FBVP) based on a shape optimization approach. Indeed, consider the following exterior FBVP: given a domain $\omega \subset \mathbb{R}^{2}$ and $Q<0$ find a domain $\Sigma$ with $\bar{\omega} \subset \Sigma$ and $u: \Sigma \backslash \bar{\omega} \rightarrow \mathbb{R}$ such that

$$
\begin{aligned}
-\Delta u=0 & & \text { on } \Sigma \backslash \bar{\omega}, \\
u=1 & & \text { on } \partial \omega, \\
u=0, \frac{\partial u}{\partial n}=Q & & \text { on } \Gamma=\partial \Sigma .
\end{aligned}
$$

One of the possibilities to solve 1.3 numerically is based on the following idea: since the system (1.3) is ill-posed due to the set of overdetermined boundary conditions on $\Gamma$, one of these conditions is realized in an optimization formulation. This results in an optimal shape design problem with an equality constraint involving a well posed state equation. Prescribing the Neumann data on $\Gamma$ one is led to the following problem:

$$
\min _{\Gamma} J(\Gamma) \equiv \frac{1}{2} \int_{\Gamma} u^{2} \mathrm{~d} \sigma,
$$

where $u$ satisfies the mixed Dirichlet-Neumann problem

$$
\begin{array}{rlrl}
-\Delta u & =0 & & \text { on } \Sigma \backslash \bar{\omega}, \\
u & =1 & & \text { on } \partial \omega, \\
\frac{\partial u}{\partial n}=Q & & \text { on } \Gamma .
\end{array}
$$

On the other hand, if the Dirichlet condition is imposed on $\Gamma$ we arrive at problem (1.1) and (1.2) with $\Omega=\Sigma \backslash \bar{\omega}, g=1$ and $\Gamma_{d}=\partial \omega$. The formulation (1.4) and (1.5) was used in [IKP] for the numerical realization of [1.3). The second order sensitivity analysis in [EH, EHS] reveals that the shape Hessian of $J$ defined by (1.1) (unlike the one of (1.4)) is coercive in the corresponding energy norm, which implies the stability of the global minimizer. Another reason to prefer the formulation (1.1) and (1.2) arises from numerical considerations: since the state problem 1.2 involves only Dirichlet data on $\partial \omega \cup \Gamma$ it can be efficiently solved by an embedding domain technique based on boundary Lagrange multipliers. It can be implemented in such a way that the Lagrange multiplier represents the normal derivative $\partial u / \partial n$ on $\partial \omega \cup \Gamma$. Thus it can be used for the evaluation of 1.2 (see [HM, HKKP1]). The shape optimization approach can be used for the numerical realization of other free boundary problems of Bernoulli type. For example in $[\overline{\mathrm{BG}}]$ the authors solved a dam problem using the cost functional (1.4). The same problem but tracking the Neumann data was considered in [HHM] using an algebraic sensitivity analysis and in [HKKP2] employing global optimization techniques for 1.1.

A formal derivation of the shape derivative of $J$ can be found in the literature (see for example $[Z]$ ). In this paper we present a rigorous justification of the shape derivative of $J$ with respect to $\Gamma$ under what we consider minimal regularity assumptions. The technique that we employ was first suggested in [IKP] and allows one to characterize the shape derivative of the mapping $\Gamma \mapsto$ $J(u(\Gamma))$ without recourse to the chain-rule approach. In particular, it does not involve the shape derivative of $u$ with respect to $\Gamma$. It will be convenient to recall the main result of [IKP] without 
entering the technical details. Consider a shape optimization problem of the form

$$
\begin{aligned}
& \min J(u, \Omega, \Gamma)=\int_{\Omega} j_{1}(u) \mathrm{d} x+\int_{\Gamma} j_{2}(u) \mathrm{d} s \\
& \text { subject to } E(u, \Omega)=0,
\end{aligned}
$$

where $E$ denotes a partial differential equation depending on a state variable $u$ and on a reference domain $\Omega$, and $\Gamma$ stands for a codimension one manifold, which may constitute a part of the boundary of $\Omega$ or lie inside of $\Omega$. Then the Eulerian derivative of $J$ at $\Omega$ in the direction $h$ is given by

$$
\mathrm{d} J(u, \Omega, \Gamma) h=-\left.\frac{\mathrm{d}}{\mathrm{d} t}\langle\tilde{E}(u, t), p\rangle\right|_{t=0}+\int_{\Omega} j_{1}(u) \operatorname{div} h \mathrm{~d} x+\int_{\Gamma} j_{2}(u) \operatorname{div}_{\Gamma} h \mathrm{~d} s .
$$

Here

$$
\mathrm{d} J(u, \Omega, \Gamma) h=\lim _{t \rightarrow 0} \frac{1}{t}\left(J\left(u_{t}, \Omega_{t}, \Gamma_{t}\right)-J(u, \Omega, \Gamma)\right),
$$

and $\tilde{E}(\cdot, t)$ is posed on the same reference domain $\Omega$ as $E$, and is related to $E$ by means of

$$
E\left(u_{t}, \Omega_{t}\right)=0 \text { if and only if } \tilde{E}\left(u^{t}, t\right)=0,
$$

where

$$
\Omega_{t}=F_{t}(\Omega), \quad u^{t}=u_{t} \circ F_{t} \quad \text { and } \quad F_{t}=\mathrm{id}+t h .
$$

As already announced, the Eulerian derivative of $J$ given in (1.7) does not depend on the shape derivative of the state. Rather it depends on the adjoint state $p$ which is the solution of the adjoint equation

$$
E_{u}^{*}(u, \Omega) p=j_{1}^{\prime}(u)+\tau_{\Gamma}^{*} j_{2}^{\prime}(u),
$$

with $\tau_{\Gamma}^{*}$ standing for the adjoint of the trace operator onto $\Gamma$.

The regularity assumptions in [IKP] require $j_{i}$ to be $C^{1,1}$ on the state space of the partial differential equation. This is not the case for the cost functional (1.1). In the present paper, however, we show that the Eulerian shape derivative of $J$ can nevertheless be obtained using the technique developed in [IKP]. Moreover, the characterization will be accomplished under the very mild $C^{1,1}$ regularity assumption on the boundary of $\Omega$.

Throughout the paper $(\cdot, \cdot)$ denotes the $L^{2}$-inner product with respect to a domain which is consistent with the context. The Euclidean product in $\mathbb{R}^{2}$ of two vectors $x$ and $y$ will be denoted by $x \cdot y$.

\section{Continuous dependence}

We embed problem (1.2) into a family of perturbed problems defined on perturbations of the reference domain $\Omega$ which are constructed by perturbing the identity. Let $U$ be a convex bounded domain of class $C^{1,1}$ such that $\bar{\Omega} \subset U$ and let

$$
\mathcal{D}=\left\{V \in C^{1,1}\left(\bar{U}, \mathbb{R}^{2}\right): V=0 \text { on } \partial U \cup \Gamma_{d}\right\}
$$


be the space of feasible deformation fields endowed with the natural norm in $C^{1,1}\left(\bar{U}, \mathbb{R}^{2}\right)$. Choose a fixed field $V \in \mathcal{D}$ and define for each $t \in \mathbb{R}$ the mapping $T_{t}: \bar{U} \rightarrow \mathbb{R}^{2}$ by

$$
T_{t}=\mathrm{id}+t V .
$$

Then $T_{t}(U)=U$ and $\left\{T_{t}\right\}$ is a family of $C^{1,1}$-diffeomorphisms for $|t|$ sufficiently small. For each $t \in \mathbb{R}$ with $|t|<\tau$ we set

$$
\Omega_{t}=T_{t}(\Omega), \quad \Gamma_{t}=T_{t}(\Gamma) .
$$

Thus $\Omega_{0}=\Omega, \Gamma_{0}=\Gamma, \bar{\Omega}_{t} \subset U$ and $\Gamma_{d}=T_{t}\left(\Gamma_{d}\right)$.

The Eulerian derivative of $J$ at $\Gamma$ in the direction of the deformation field $V$ is defined as

$$
\mathrm{d} J(\Gamma) V=\lim _{t \rightarrow 0} \frac{1}{t}\left(J\left(\Gamma_{t}\right)-J(\Gamma)\right),
$$

where

$$
J\left(\Gamma_{t}\right)=\frac{1}{2} \int_{\Gamma_{t}}\left(\frac{\partial u_{t}}{\partial n_{t}}-Q\right)^{2} \mathrm{~d} \sigma_{t} .
$$

Above $n_{t}$ stands for the outer normal unit vector to $\Gamma_{t}$ and $u_{t}$ is the solution of the perturbed Dirichlet problem

$$
\begin{aligned}
-\Delta u_{t}=0 & \text { on } \Omega_{t}, \\
u_{t}=g & \text { on } \Gamma_{d}, \\
u_{t}=0 & \text { on } \Gamma_{t} .
\end{aligned}
$$

The functional $J$ is called shape differentiable at $\Gamma$ if $\mathrm{d} J(\Gamma) V$ exists for all $V \in \mathcal{D}$ and defines a continuous linear functional on $\mathcal{D}$. For an in-depth discussion of these concepts we refer to [DZ, SZ].

The variational form of 2.3 is given by:

Find $u_{t} \in H^{1}\left(\Omega_{t}\right)$ such that $u_{t}=g$ on $\Gamma_{d}, u_{t}=0$ on $\Gamma_{t}$ and

$$
\left(\nabla u_{t}, \nabla v_{t}\right)=0 \quad \text { for all } v_{t} \in H_{0}^{1}\left(\Omega_{t}\right) .
$$

It is known that (2.4) has a unique solution $u_{t}$ which in addition satisfies $u_{t} \in H^{2}\left(\Omega_{t}\right)$. For $t=0$ equation (2.4) represents the weak form of the reference problem [1.2]. Any function $\varphi_{t}: \Omega_{t} \rightarrow \mathbb{R}$ can be referred to the reference domain by

$$
\varphi^{t}=\varphi_{t} \circ T_{t}: \Omega \rightarrow \mathbb{R} .
$$

From the chain rule it follows that the gradients of $\varphi_{t}$ and $\varphi^{t}$ are related by

$$
\nabla \varphi_{t} \circ T_{t}=\left(D T_{t}\right)^{-T} \nabla \varphi^{t},
$$

where $D f$ stands for the derivative of the mapping $f$. Let $u_{t}$ denote the solution of 2.4 . Then $u^{t}=u_{t} \circ T_{t}$ satisfies the transformed equation in $\Omega$ :

$$
\left(A(t) \nabla u^{t}, \nabla \psi\right)=0
$$


for all $\psi \in H_{0}^{1}(\Omega), u^{t}=0$ on $\Gamma$ and $u^{t}=g$ on $\Gamma_{d}$. Here and in the following we use the notation

$$
I_{t}=\operatorname{det} D T_{t}, \quad A(t)=\left(D T_{t}\right)^{-1}\left(D T_{t}\right)^{-T} I_{t}, \quad w_{t}=I_{t}\left|\left(D T_{t}\right)^{-T} n\right|,
$$

where $n$ denotes the outer normal unit vector to $\partial \Omega$ and $|\cdot|$ is the Euclidean norm of a vector in $\mathbb{R}^{2}$ or the spectral norm of a matrix in $\mathbb{R}^{2 \times 2}$. By an induction argument one can prove the expansion

$$
I_{t}(x)=1+t \operatorname{div} V(x)+t^{2} \sum_{i=0}^{n-2} t^{i} f_{i}(D V(x)), \quad x \in \bar{U},
$$

with suitable polynomials $f_{i}$. This implies that for any $0<\alpha<1$, the inequality

$$
I_{t}(x) \geqslant \alpha>0, \quad x \in \bar{U},
$$

holds for $|t|<\tau$, with $\tau>0$ sufficiently small. If, in addition, $\tau|D V|_{\infty} \equiv \tau \max \left\{\left|\partial_{x_{j}} V_{i}\right|: 1 \leqslant\right.$ $i, j \leqslant 2, x \in \bar{U}\}<1$ then the inverse of the Jacobian $D T_{t}$ can be represented by the Neumann series

$$
\left(D T_{t}\right)^{-1}=\sum_{i=0}^{\infty}(-t)^{i}(D V)^{i}, \quad|t|<\tau,
$$

which converges uniformly for $(t, x) \in[-\tau, \tau] \times \bar{U}$. For further reference we note the bound

$$
\left|\left(D T_{t}\right)^{-1}\right|_{\infty} \leqslant \frac{1}{1-\tau|D V|_{\infty}} .
$$

Let $\mathcal{J}$ denote the interval $[-\tau, \tau]$. Then the following regularity properties of the transformation $T_{t}$ can be shown ([DZ, [KP] $)$ :

$$
\begin{array}{lll}
T_{0}=\mathrm{id}, & t \mapsto T_{t} \in C^{1}\left(\mathcal{J}, C^{1,1}\left(\bar{U}, \mathbb{R}^{2}\right)\right), \\
t \mapsto T_{t}^{-1} \in C\left(\mathcal{J}, C^{1}\left(\bar{U}, \mathbb{R}^{2}\right)\right), & t \mapsto I_{t} \in C^{1}(\mathcal{J}, C(\bar{U})), \\
t \mapsto D\left(T_{t}\right)^{-T} \in C\left(\mathcal{J}, C\left(\bar{U}, \mathbb{R}^{2 \times 2}\right)\right), & t \mapsto w_{t} \in C(\mathcal{J}, C(\Gamma)), \\
\left.\frac{\mathrm{d}}{\mathrm{d} t} T_{t}\right|_{t=0}=V, & \left.\frac{\mathrm{d}}{\mathrm{d} t} T_{t}^{-1}\right|_{t=0}=-V, \\
\left.\frac{\mathrm{d}}{\mathrm{d} t} D T_{t}\right|_{t=0}=D V, & \left.\frac{\mathrm{d}}{\mathrm{d} t} D T_{t}^{-1}\right|_{t=0}=-D V, \\
\left.\frac{\mathrm{d}}{\mathrm{d} t} I_{t}\right|_{t=0}=\operatorname{div} V, & \left.\frac{\mathrm{d}}{\mathrm{d} t} w_{t}\right|_{t=0}=\operatorname{div}_{\Gamma} V .
\end{array}
$$

The limits defining the derivatives at $t=0$ exist uniformly in $x \in \bar{U}$. The surface divergence $\operatorname{div}_{\Gamma}$ is defined for $\varphi \in C^{1}\left(\bar{U}, \mathbb{R}^{2}\right)$ by

$$
\operatorname{div}_{\Gamma} \varphi:=\left.\operatorname{div} \varphi\right|_{\Gamma}-(D \varphi n) \cdot n .
$$

Furthermore, using 2.8 the estimate

$$
(A(t) \xi, \xi)=\left(D\left(T_{t}\right)^{-T} \xi, I_{t} D\left(T_{t}\right)^{-T} \xi\right) \geqslant \alpha\left|D\left(T_{t}\right)^{-T} \xi\right|^{2} \geqslant \frac{\alpha}{\left|D\left(T_{t}\right)^{T}\right|^{2}}|\xi|^{2} \geqslant \frac{\alpha}{2}|\xi|^{2}
$$

follows for $(\xi, x) \in \mathbb{R}^{2} \times \Omega$ and $\tau$ sufficiently small, which ensures that the bilinear form in 2.6 is elliptic uniformly in $t \in \mathcal{J}$. 
THEOREM 2.1 The solution $u^{t}$ of equation 2.6 satisfies $u^{t} \in H^{2}(\Omega)$ for $t$ sufficiently small. Moreover,

$$
\lim _{t \rightarrow 0} \frac{1}{\sqrt{|t|}}\left|u^{t}-u\right|_{H^{2}(\Omega)}=0,
$$

where $u$ is the weak solution of (1.2).

Proof. It suffices to show the second statement. Subtracting (2.4) with $t=0$ from 2.6) one finds that $\frac{1}{t}\left(u^{t}-u\right)$ satisfies

$$
\left(A(t) \nabla\left(\frac{1}{t}\left(u^{t}-u\right)\right), \nabla \psi\right)=-\left(\frac{1}{t}(A(t)-I) \nabla u, \nabla \psi\right)=\left(\operatorname{div}\left(\frac{1}{t}(A(t)-I) \nabla u\right), \psi\right)
$$

for every $\psi$ in $H_{0}^{1}(\Omega)$. Since $\frac{1}{t}\left(u^{t}-u\right)$ belongs to $H_{0}^{1}(\Omega)$ standard elliptic regularity theory implies $u^{t}-u \in H^{2}(\Omega) \cap H_{0}^{1}(\Omega)$ and

$$
\left|\frac{1}{t}\left(u^{t}-u\right)\right|_{H^{2}(\Omega)} \leqslant c\left|\operatorname{div}\left(\frac{1}{t}(A(t)-I) \nabla u\right)\right|_{L^{2}(\Omega)}
$$

with a constant $c>0$ which depends only on $|A(t)|_{\infty}$ and the Lipschitz constant for $D V$. This may be chosen independently of $t$ (see Theorem IV-2.1 in $[\mathrm{Ne}]$ and the proof of Theorem 6.3.4 in [E] which is applicable to an equation on a $C^{1,1}$-domain with $C^{0,1}$-coefficients). The result then follows if we bound $\left|\frac{1}{t} D(A(t)-I)\right|_{\infty}$ uniformly in $t$. For this purpose consider any partial derivative $\partial_{k}$, $k=1,2$ :

$$
\partial_{k} \frac{1}{t}(A(t)-I)=\frac{1}{t} \partial_{k}\left(D T_{t}^{-1}-I\right) D T_{t}^{-T} I_{t}+\frac{1}{t} \partial_{k}\left(D T_{t}^{-T}-I\right) I_{t}+\frac{1}{t} \partial_{k}\left(I_{t}-1\right) I
$$

and observe that

$$
\begin{aligned}
\frac{1}{t} \partial_{k}\left(D T_{t}^{-1}-I\right) & =\frac{1}{t} \partial_{k}\left(D T_{t}^{-1}\left(I-D T_{t}\right)\right)=-\partial_{k}\left(D T_{t}^{-1} D V\right) \\
& =t\left(D T_{t}\right)^{-1}\left(\partial_{k} D V\right)\left(D T_{t}\right)^{-1} D V-D T_{t}^{-1} \partial_{k} D V
\end{aligned}
$$

By Rademacher's theorem $\partial_{k} D V$ exists almost everywhere in $U$ and is bounded by the Lipschitz constant for $D V$. A bound uniform in $t$ follows from $(2.9)$. The estimate for $\partial_{k} \frac{1}{t}\left(I_{t}-1\right)$ is similar and uses (2.7).

The above proof actually shows that the difference quotient $\frac{1}{t}\left|u^{t}-u\right|_{H^{2}(\Omega)}$ is bounded as $t \rightarrow 0$.

COROLlaRY 2.1 Let $u_{t}$ and $u$ stand for the weak solution of (2.4), respectively (1.2). Then

$$
\lim _{t \rightarrow 0} \frac{1}{\sqrt{|t|}}\left|\frac{\partial u_{t}}{\partial n_{t}} \circ T_{t}-\frac{\partial u}{\partial n}\right|_{L^{2}(\Gamma)}=0 .
$$

Proof. Define $n^{t}=n_{t} \circ T_{t}$. Then

$$
n^{t}=\frac{D T_{t}^{-T} n}{\left|D T_{t}^{-T} n\right|}=\frac{I_{t}}{w_{t}} D T_{t}^{-T} n
$$

([DZ, Theorem 4.4]), which is meaningful because $w_{t} \rightarrow 1$ uniformly on $\Gamma$. As a consequence we obtain 


$$
\begin{aligned}
\frac{\partial u_{t}}{\partial n_{t}} \circ T_{t}-\frac{\partial u}{\partial n} & =\nabla u_{t} \circ T_{t} \cdot n_{t} \circ T_{t}-\nabla u \cdot n=D T_{t}^{-T} \nabla u^{t} \cdot n^{t}-\nabla u \cdot n \\
& =\frac{1}{w_{t}} D T_{t}^{-T} \nabla u^{t} \cdot I_{t} D T_{t}^{-T} n-\nabla u \cdot n=\frac{1}{w_{t}}\left(A(t) \nabla u^{t}-w_{t} \nabla u\right) \cdot n \\
& =\frac{1}{w_{t}}\left((A(t)-I) \nabla u^{t} \cdot n+\frac{\partial}{\partial n}\left(u^{t}-u\right)-\left(w_{t}-1\right) \frac{\partial u}{\partial n}\right) .
\end{aligned}
$$

Now the result follows by using the differentiability of $t \mapsto A(t)$ and $t \mapsto w_{t}$ at $t=0$, the trace theorem and Theorem 2.1

\section{Sensitivity analysis}

In order to make the paper self-contained we state the main theoretical tools which will be used in the analysis below.

LEMMA 3.1 ([|MS|) If $\varphi_{t} \in L^{1}\left(\Gamma_{t}\right)$ then $\varphi_{t} \circ T_{t} \in L^{1}(\Gamma)$ and

$$
\int_{\Gamma_{t}} \varphi_{t} \mathrm{~d} \sigma_{t}=\int_{\Gamma} \varphi_{t} \circ T_{t} w_{t} \mathrm{~d} \sigma
$$

LEMMA $3.2([\mathrm{MS}, \mathrm{DZ}])$ Let $f \in C\left((-\tau, \tau), W^{1,1}(U)\right)$ and assume that $\frac{\partial f}{\partial t}(0, \cdot)$ exists in $L^{1}(U)$. Then

$$
\left.\frac{\mathrm{d}}{\mathrm{d} t} \int_{\Omega_{t}} f(t, x) \mathrm{d} x_{t}\right|_{t=0}=\int_{\Omega} \frac{\partial f}{\partial t}(0, x) \mathrm{d} x+\int_{\Gamma} f(0, x) V \cdot n \mathrm{~d} \sigma .
$$

LEMMA 3.3 (Tangential Green's formula) If $f \in W^{2,1}(U)$, then

$$
\int_{\Gamma}\left(f \operatorname{div}_{\Gamma} V+\nabla_{\Gamma} f \cdot V\right) \mathrm{d} \sigma=\int_{\Gamma} \kappa f V \cdot n \mathrm{~d} \sigma,
$$

where $\kappa$ denotes the curvature of $\Gamma$ and the tangential gradient $\nabla_{\Gamma}$ is given by

$$
\nabla_{\Gamma} f=\left.\nabla f\right|_{\Gamma}-\frac{\partial f}{\partial n} n
$$

A proof of the tangential Green's formula can be found in [MS] (referred to as surface integration by parts formula) where it is also shown that the curvature of a $C^{1,1}$-domain makes sense in $L^{\infty}(\Gamma)$.

Since $\Omega \in C^{1,1}$ and $g \in H^{3 / 2}\left(\Gamma_{d}\right)$, the solution $u$ of the reference problem 1.2 belongs to $H^{2}(\Omega)$. Define the adjoint equation

$$
\begin{aligned}
-\Delta p & =0 & & \text { in } \Omega, \\
p & =0 & & \text { on } \Gamma_{d}, \\
p & =\frac{\partial u}{\partial n}-Q & & \text { on } \Gamma,
\end{aligned}
$$

or equivalently

$$
(\nabla p, \nabla \psi)=0 \quad \text { for all } \psi \in H_{0}^{1}(\Omega),
$$

$p=0$ on $\Gamma_{d}$ and $p=\partial u / \partial n-Q$ on $\Gamma$. Since $\partial u / \partial n-Q \in H^{1 / 2}(\Gamma)$ equation 3.2 has a unique solution $p \in H^{1}(\Omega)$.

Now we turn to the shape differentiability of the cost functional (1.1). 
THEOREM 3.1 Let $\Omega \in C^{1,1}, g \in H^{3 / 2}\left(\Gamma_{d}\right)$ and $\mathcal{D} \subset C^{1,1}\left(\bar{U}, \mathbb{R}^{2}\right)$ be defined by 2.1$)$. Then the cost functional

$$
J(\Gamma)=\frac{1}{2} \int_{\Gamma}\left(\frac{\partial u}{\partial n}-Q\right)^{2} \mathrm{~d} \sigma
$$

is shape differentiable. For every $V \in \mathcal{D}$ its derivative in the direction $V$ is given by

$$
\mathrm{d} J(\Gamma) V=-\int_{\Gamma}\left(\frac{1}{2} p^{2}+Q p\right) \kappa V \cdot n \mathrm{~d} \sigma-\left\langle\frac{\partial p}{\partial n}, \frac{\partial u}{\partial n} V \cdot n\right\rangle_{\Gamma},
$$

where $u$ and $p$ stand for the solution of (1.2), respectively (3.2), and $\langle\cdot, \cdot\rangle_{\Gamma}$ denotes the duality pairing between $H^{-1 / 2}(\Gamma)$ and $H^{1 / 2}(\Gamma)$.

Proof. It suffices to show that the Eulerian derivative of $J$ at $\Gamma$ in the direction $V$ can be represented in the form (3.3). Transforming the boundary integral according to Lemma 3.1 we obtain

$$
J\left(\Gamma_{t}\right)=\frac{1}{2} \int_{\Gamma_{t}}\left(\frac{\partial u_{t}}{\partial n_{t}}-Q\right)^{2} \mathrm{~d} \sigma_{t}=\frac{1}{2} \int_{\Gamma}\left(\left(\nabla u_{t} \cdot n_{t}\right) \circ T_{t}-Q\right)^{2} w_{t} \mathrm{~d} \sigma .
$$

In order to calculate the Eulerian derivative of $J$ we exploit the structure of $J$ using the identity $a^{2}-b^{2}=2(a-b) b+(a-b)^{2}$ :

$$
\begin{aligned}
J\left(\Gamma_{t}\right)-J(\Gamma)= & \frac{1}{2} \int_{\Gamma}\left(w_{t}-1\right)\left[\left(\frac{\partial u_{t}}{\partial n_{t}} \circ T_{t}-Q\right)^{2}-\left(\frac{\partial u}{\partial n}-Q\right)^{2}\right] \mathrm{d} \sigma \\
& +\frac{1}{2} \int_{\Gamma}\left(w_{t}-1\right)\left(\frac{\partial u}{\partial n}-Q\right)^{2} \mathrm{~d} \sigma+\frac{1}{2} \int_{\Gamma}\left[\left(\frac{\partial u_{t}}{\partial n_{t}} \circ T_{t}-Q\right)^{2}-\left(\frac{\partial u}{\partial n}-Q\right)^{2}\right] \mathrm{d} \sigma \\
= & \frac{1}{2} \int_{\Gamma}\left(w_{t}-1\right)\left(\frac{\partial u_{t}}{\partial n_{t}} \circ T_{t}-\frac{\partial u}{\partial n}\right)\left(\frac{\partial u_{t}}{\partial n_{t}} \circ T_{t}+\frac{\partial u}{\partial n}-2 Q\right) \mathrm{d} \sigma \\
& +\frac{1}{2} \int_{\Gamma}\left(w_{t}-1\right)\left(\frac{\partial u}{\partial n}-Q\right)^{2} \mathrm{~d} \sigma \\
& +\int_{\Gamma}\left(\left(\nabla u_{t} \cdot n_{t}\right) \circ T_{t}-\frac{\partial u}{\partial n}\right)\left(\frac{\partial u}{\partial n}-Q\right) \mathrm{d} \sigma+\frac{1}{2} \int_{\Gamma}\left(\frac{\partial u_{t}}{\partial n_{t}} \circ T_{t}-\frac{\partial u}{\partial n}\right)^{2} \mathrm{~d} \sigma \\
\equiv & J_{1}(t)+J_{2}(t)+J_{3}(t)+J_{4}(t) .
\end{aligned}
$$

By the last equality in 2.10 and Corollary 2.1 we deduce

$$
\begin{aligned}
& \dot{J}_{1}(0)=\dot{J}_{4}(0)=0 \\
& \dot{J}_{2}(0)=\frac{1}{2} \lim _{t \rightarrow 0} \int_{\Gamma} \frac{1}{t}\left(w_{t}-1\right)\left(\frac{\partial u}{\partial n}-Q\right)^{2} \mathrm{~d} \sigma=\frac{1}{2} \int_{\Gamma}\left(\frac{\partial u}{\partial n}-Q\right)^{2} \operatorname{div}_{\Gamma} V \mathrm{~d} \sigma .
\end{aligned}
$$

The treatment of $J_{3}(t)$ is more delicate. Reasoning as in the proof of Corollary 2.1 one obtains

$$
\int_{\Gamma}\left(\nabla u_{t} \cdot n_{t}\right) \circ T_{t}\left(\frac{\partial u}{\partial n}-Q\right) \mathrm{d} \sigma=\int_{\Gamma} A(t) \nabla u^{t} \cdot n \frac{1}{w_{t}}\left(\frac{\partial u}{\partial n}-Q\right) \mathrm{d} \sigma,
$$

which suggests writing $J_{3}$ in the following form: 


$$
\begin{aligned}
J_{3}(t)= & \left(\int_{\Gamma}\left(A(t) \nabla u^{t} \cdot n\right)\left(\frac{\partial u}{\partial n}-Q\right) \mathrm{d} \sigma-\int_{\Gamma} \frac{\partial u}{\partial n}\left(\frac{\partial u}{\partial n}-Q\right) \mathrm{d} \sigma\right) \\
& +\int_{\Gamma}\left(A(t) \nabla u^{t} \cdot n\right)\left(\frac{\partial u}{\partial n}-Q\right)\left(w_{t}^{-1}-1\right) \mathrm{d} \sigma=: J_{31}(t)+J_{32}(t) .
\end{aligned}
$$

Using Theorem 2.1 together with $\lim _{t \rightarrow 0} A(t)=I$ and $\lim _{t \rightarrow 0} w_{t}=1$ uniformly on $\Gamma$ and $J_{32}(0)=0$ we obtain

$$
\begin{aligned}
\dot{J}_{32}(0) & =\lim _{t \rightarrow 0} \int_{\Gamma}(A(t)-I) \nabla u^{t} \cdot n\left(\frac{\partial u}{\partial n}-Q\right) \frac{1-w_{t}}{t w_{t}} \mathrm{~d} \sigma+\lim _{t \rightarrow 0} \int_{\Gamma} \frac{\partial u^{t}}{\partial n}\left(\frac{\partial u}{\partial n}-Q\right) \frac{1-w_{t}}{t w_{t}} \mathrm{~d} \sigma \\
& =-\int_{\Gamma} \frac{\partial u}{\partial n}\left(\frac{\partial u}{\partial n}-Q\right) \operatorname{div}_{\Gamma} V \mathrm{~d} \sigma .
\end{aligned}
$$

Using the adjoint state $p$ one can write $J_{31}(t)$ as

$$
J_{31}(t)=\int_{\Gamma} A(t) \nabla u^{t} \cdot n p \mathrm{~d} \sigma-\int_{\Gamma} \frac{\partial u}{\partial n} p \mathrm{~d} \sigma .
$$

Then Green's formula and the strong form of 2.6 imply

$$
\int_{\Gamma} A(t) \nabla u^{t} \cdot n p \mathrm{~d} \sigma=\left(\operatorname{div}\left(A(t) \nabla u^{t}\right), p\right)+\left(A(t) \nabla u^{t}, \nabla p\right)=\left(A(t) \nabla u^{t}, \nabla p\right)
$$

and analogously

$$
\int_{\Gamma} \frac{\partial u}{\partial n} p \mathrm{~d} \sigma=(\nabla u, \nabla p)
$$

From this we derive

$$
\begin{aligned}
J_{31}(t) & =\left(A(t) \nabla u^{t}, \nabla p\right)-(\nabla u, \nabla p) \\
& =\left(A(t) \nabla\left(u^{t}-u\right), \nabla p\right)+(A(t) \nabla u, \nabla p)-(\nabla u, \nabla p) \\
& =\left((A(t)-I) \nabla\left(u^{t}-u\right), \nabla p\right)+\left(\nabla\left(u^{t}-u\right), \nabla p\right)+(A(t) \nabla u, \nabla p)-(\nabla u, \nabla p) \\
& =\left((A(t)-I) \nabla\left(u^{t}-u\right), \nabla p\right)+(A(t) \nabla u, \nabla p)-(\nabla u, \nabla p),
\end{aligned}
$$

where we have used the fact that $u^{t}-u \in H_{0}^{1}(\Omega)$ and the adjoint equation (3.2). Taking the derivative of $J_{31}$ is complicated by the fact that $p$ belongs to $H^{1}(\Omega)$ only. To overcome this difficulty we choose a sequence $\left(u_{k}\right)_{k=1}^{\infty} \subset C^{\infty}(\bar{\Omega})$ which approximates $u$ in $H^{2}(\Omega)$ and denote by $p_{k}$ the solution of the adjoint equation (3.1) with $\partial u / \partial n-Q$ replaced by $\partial u_{k} / \partial n-Q$. Then $p_{k} \in H^{2}(\Omega)$ and $\lim _{k \rightarrow \infty} p_{k}=p$ in $H^{1}(\Omega)$. By differentiability of $t \mapsto A(t)$ and $t \mapsto u^{t}$ at $t=0$ we find

$$
\lim _{t \rightarrow 0}\left(\frac{1}{t}(A(t)-I) \nabla\left(u^{t}-u\right), \nabla p\right)=0 .
$$

This leads to

$$
\begin{aligned}
\dot{J}_{31}(0) & =\lim _{t \rightarrow 0} \frac{1}{t}[(A(t) \nabla u, \nabla p)-(\nabla u, \nabla p)]=\lim _{t \rightarrow 0} \lim _{k \rightarrow \infty} \frac{1}{t}\left[\left(A(t) \nabla u_{k}, \nabla p_{k}\right)-\left(\nabla u_{k}, \nabla p_{k}\right)\right] \\
& =\lim _{k \rightarrow \infty} \lim _{t \rightarrow 0} \frac{1}{t}\left[\left(A(t) \nabla u_{k}, \nabla p_{k}\right)-\left(\nabla u_{k}, \nabla p_{k}\right)\right] \\
& =\left.\lim _{k \rightarrow \infty} \frac{\mathrm{d}}{\mathrm{d} t} \int_{\Omega_{t}} \nabla\left(u_{k} \circ T_{t}^{-1}\right) \cdot \nabla\left(p_{k} \circ T_{t}^{-1}\right) \mathrm{d} x\right|_{t=0} \cdot
\end{aligned}
$$


The interchange of the two limits above is justified because $\lim _{t \rightarrow 0} \frac{1}{t}(A(t)-I)$ exists uniformly in $x \in \bar{U}$. If we extend $u_{k}$ and $p_{k}$ to elements in $H^{2}(U)$, the last derivative can be calculated using Lemma 3.2 and $\left.\frac{\mathrm{d}}{\mathrm{d} t} T_{t}^{-1}\right|_{t=0}=-V$ :

$$
\dot{J}_{31}(0)=\lim _{k \rightarrow \infty}\left[\int_{\Gamma} \nabla u_{k} \cdot \nabla p_{k} V \cdot n \mathrm{~d} \sigma+\left(\nabla\left(-\nabla u_{k} \cdot V\right), \nabla p_{k}\right)+\left(\nabla u_{k}, \nabla\left(-\nabla p_{k} \cdot V\right)\right)\right] \text {. }
$$

Further Green's formula combined with $\Delta p_{k}=0$ in $\Omega$ and $V=0$ on $\Gamma_{d}$ leads to

$$
\begin{aligned}
\dot{J}_{31}(0)= & \lim _{k \rightarrow \infty}\left[\int_{\Gamma} \nabla u_{k} \cdot \nabla p_{k} V \cdot n \mathrm{~d} \sigma+\left(\nabla u_{k} \cdot V, \Delta p_{k}\right)-\int_{\Gamma} \frac{\partial p_{k}}{\partial n} \nabla u_{k} \cdot V \mathrm{~d} \sigma\right. \\
& \left.+\left(\Delta u_{k}, \nabla p_{k} \cdot V\right)-\int_{\Gamma} \frac{\partial u_{k}}{\partial n} \nabla p_{k} \cdot V \mathrm{~d} \sigma\right] \\
= & \lim _{k \rightarrow \infty}\left[\int_{\Gamma} \nabla u_{k} \cdot \nabla p_{k} V \cdot n \mathrm{~d} \sigma-\int_{\Gamma} \frac{\partial p_{k}}{\partial n} \nabla u_{k} \cdot V \mathrm{~d} \sigma\right. \\
& \left.-\int_{\Gamma} \frac{\partial u_{k}}{\partial n} \nabla p_{k} \cdot V \mathrm{~d} \sigma+\left(\Delta u_{k}, \nabla p_{k} \cdot V\right)\right] .
\end{aligned}
$$

Collecting (3.4)-3.7) leads to

$$
\begin{aligned}
\mathrm{d} J(\Gamma) V= & \dot{J}_{1}(0)+\dot{J}_{2}(0)+\dot{J}_{3}(0)+\dot{J}_{4}(0) \\
= & \frac{1}{2} \int_{\Gamma}\left(\frac{\partial u}{\partial n}-Q\right)^{2} \operatorname{div}_{\Gamma} V \mathrm{~d} \sigma-\int_{\Gamma} \frac{\partial u}{\partial n}\left(\frac{\partial u}{\partial n}-Q\right) \operatorname{div}_{\Gamma} V \mathrm{~d} \sigma \\
& +\lim _{k \rightarrow \infty}\left[\int_{\Gamma} \nabla u_{k} \cdot \nabla p_{k} V \cdot n \mathrm{~d} \sigma-\int_{\Gamma} \frac{\partial p_{k}}{\partial n} \nabla u_{k} \cdot V \mathrm{~d} \sigma\right. \\
& \left.-\int_{\Gamma} \frac{\partial u_{k}}{\partial n} \nabla p_{k} \cdot V \mathrm{~d} \sigma+\left(\Delta u_{k}, \nabla p_{k} \cdot V\right)\right] \\
= & \lim _{k \rightarrow \infty}\left[\frac{1}{2} \int_{\Gamma}\left(\frac{\partial u_{k}}{\partial n}-Q\right)^{2} \operatorname{div}_{\Gamma} V \mathrm{~d} \sigma-\int_{\Gamma} \frac{\partial u_{k}}{\partial n}\left(\frac{\partial u_{k}}{\partial n}-Q\right) \operatorname{div}_{\Gamma} V \mathrm{~d} \sigma\right. \\
& +\int_{\Gamma} \nabla u_{k} \cdot \nabla p_{k} V \cdot n \mathrm{~d} \sigma-\int_{\Gamma} \frac{\partial p_{k}}{\partial n} \nabla u_{k} \cdot V \mathrm{~d} \sigma \\
& \left.-\int_{\Gamma} \frac{\partial u_{k}}{\partial n} \nabla p_{k} \cdot V \mathrm{~d} \sigma+\left(\Delta u_{k}, \nabla p_{k} \cdot V\right)\right] \\
= & \lim _{k \rightarrow \infty}\left[-\int_{\Gamma}\left(\frac{1}{2} p_{k}^{2}+Q p_{k}\right) \operatorname{div}_{\Gamma} V \mathrm{~d} \sigma+\int_{\Gamma} \nabla u_{k} \cdot \nabla p_{k} V \cdot n \mathrm{~d} \sigma\right. \\
& \left.-\int_{\Gamma} \frac{\partial p_{k}}{\partial n} \nabla u_{k} \cdot V \mathrm{~d} \sigma-\int_{\Gamma} \frac{\partial u_{k}}{\partial n} \nabla p_{k} \cdot V \mathrm{~d} \sigma\right]
\end{aligned}
$$

where we have used the equalities $p_{k}=\frac{\partial u_{k}}{\partial n}-Q$ on $\Gamma$ and $\lim _{k \rightarrow \infty} \Delta u_{k}=0$ in $L^{2}(\Omega)$ in the last step. Next we rewrite the first term using the tangential Green's formula (Lemma 3.3 ) and the definition of the tangential gradient $\nabla_{\Gamma} p_{k}$. This results in 


$$
\begin{aligned}
-\int_{\Gamma}\left(\frac{1}{2} p_{k}^{2}+Q p_{k}\right) & \operatorname{div}_{\Gamma} V \mathrm{~d} \sigma=\int_{\Gamma} \nabla_{\Gamma}\left(\frac{1}{2} p_{k}^{2}+Q p_{k}\right) \cdot V \mathrm{~d} \sigma-\int_{\Gamma}\left(\frac{1}{2} p_{k}^{2}+Q p_{k}\right) \kappa V \cdot n \mathrm{~d} \sigma \\
& =\int_{\Gamma}\left(p_{k}+Q\right) \nabla_{\Gamma} p_{k} \cdot V \mathrm{~d} \sigma-\int_{\Gamma}\left(\frac{1}{2} p_{k}^{2}+Q p_{k}\right) \kappa V \cdot n \mathrm{~d} \sigma \\
& =\int_{\Gamma} \frac{\partial u_{k}}{\partial n} \nabla p_{k} \cdot V \mathrm{~d} \sigma-\int_{\Gamma} \frac{\partial u_{k}}{\partial n} \frac{\partial p_{k}}{\partial n} V \cdot n \mathrm{~d} \sigma-\int_{\Gamma}\left(\frac{1}{2} p_{k}^{2}+Q p_{k}\right) \kappa V \cdot n \mathrm{~d} \sigma .
\end{aligned}
$$

This entails

$$
\begin{aligned}
d J(\Gamma) V= & \lim _{k \rightarrow \infty}\left[\int_{\Gamma} \frac{\partial u_{k}}{\partial n} \nabla p_{k} \cdot V \mathrm{~d} \sigma-\int_{\Gamma} \frac{\partial u_{k}}{\partial n} \frac{\partial p_{k}}{\partial n} V \cdot n \mathrm{~d} \sigma-\int_{\Gamma}\left(\frac{1}{2} p_{k}^{2}+Q p_{k}\right) \kappa V \cdot n \mathrm{~d} \sigma\right. \\
& \left.+\int_{\Gamma} \nabla u_{k} \cdot \nabla p_{k} V \cdot n \mathrm{~d} \sigma-\int_{\Gamma} \frac{\partial p_{k}}{\partial n} \nabla u_{k} \cdot V \mathrm{~d} \sigma-\int_{\Gamma} \frac{\partial u_{k}}{\partial n} \nabla p_{k} \cdot V \mathrm{~d} \sigma\right] \\
= & \lim _{k \rightarrow \infty}\left[-\int_{\Gamma}\left(\frac{1}{2} p_{k}^{2}+Q p_{k}\right) \kappa V \cdot n \mathrm{~d} \sigma-\int_{\Gamma} \frac{\partial u_{k}}{\partial n} \frac{\partial p_{k}}{\partial n} V \cdot n \mathrm{~d} \sigma\right. \\
& \left.+\int_{\Gamma} \nabla u_{k} \cdot \nabla p_{k} V \cdot n \mathrm{~d} \sigma-\int_{\Gamma} \frac{\partial p_{k}}{\partial n} \nabla u_{k} \cdot V \mathrm{~d} \sigma\right] \\
= & -\int_{\Gamma}\left(\frac{1}{2} p^{2}+Q p\right) \kappa V \cdot n \mathrm{~d} \sigma-\lim _{k \rightarrow \infty} \int_{\Gamma} \frac{\partial p_{k}}{\partial n} \frac{\partial u_{k}}{\partial n} V \cdot n \mathrm{~d} \sigma \\
& +\lim _{k \rightarrow \infty}\left[\int_{\Gamma} \frac{\partial u_{k}}{\partial n} \frac{\partial p_{k}}{\partial n} V \cdot n \mathrm{~d} \sigma+\int_{\Gamma} \nabla u_{k} \cdot \tau \nabla p_{k} \cdot \tau V \cdot n \mathrm{~d} \sigma\right. \\
& \left.-\int_{\Gamma} \frac{\partial u_{k}}{\partial n} \frac{\partial p_{k}}{\partial n} V \cdot n \mathrm{~d} \sigma-\int_{\Gamma} \frac{\partial p_{k}}{\partial n} \nabla u_{k} \cdot \tau V \cdot \tau \mathrm{d} \sigma\right] \\
= & -\int_{\Gamma}\left(\frac{1}{2} p^{2}+Q p\right) \kappa V \cdot n \mathrm{~d} \sigma-\lim _{k \rightarrow \infty} \int_{\Gamma} \frac{\partial p_{k}}{\partial n} \frac{\partial u_{k}}{\partial n} V \cdot n \mathrm{~d} \sigma \\
& +\lim _{k \rightarrow \infty}\left[\int_{\Gamma}\left(\nabla p_{k} \cdot \tau\right)\left(\nabla u_{k} \cdot \tau\right) V \cdot n \mathrm{~d} \sigma-\int_{\Gamma} \frac{\partial p_{k}}{\partial n}\left(\nabla u_{k} \cdot \tau\right) V \cdot \tau \mathrm{d} \sigma\right]
\end{aligned}
$$

where $\tau$ denotes the properly oriented tangential unit vector. Since $\partial u_{k} / \partial n, \partial u / \partial n \in H^{1 / 2}(\Gamma)$ there exist their respective extensions $w_{k}, w \in H^{1}(\Omega)$ such that $w_{k} \rightarrow w$ in $H^{1}(\Omega)$ and the normal field $n \in C^{0,1}(\Gamma)$ can be extended to a vector field $\mathcal{N} \in C^{0,1}(\bar{\Omega})[\overline{\mathrm{GR}}]$. Then it is readily seen that $w_{k} V \cdot \mathcal{N}, w V \cdot \mathcal{N} \in H^{1}(\Omega)$ and $w_{k} V \cdot \mathcal{N}$ converges to $w V \cdot \mathcal{N}$ in $H^{1}(\Omega)$ and hence $\left(\partial u_{k} / \partial n\right) V \cdot n$ converges to $(\partial u / \partial n) V \cdot n$ in $H^{1 / 2}(\Gamma)$. Since $\operatorname{div}\left(\nabla p_{k}\right)=\operatorname{div}(\nabla p)=0$ in $\Omega$, we see that $\nabla p_{k}$ converges to $\nabla p$ not only in $L^{2}(\Omega)^{2}$ but also in $H$ (div, $\Omega$ ). Therefore the normal derivative can be extended to a bounded linear operator in $\mathcal{L}\left(H(\operatorname{div}, \Omega), H^{-1 / 2}(\Gamma)\right)$ [GR, Theorem 2.5], enabling us to pass to the limit

$$
\lim _{k \rightarrow \infty} \int_{\Gamma} \frac{\partial p_{k}}{\partial n} \frac{\partial u_{k}}{\partial n} V \cdot n \mathrm{~d} \sigma=\left\langle\frac{\partial p}{\partial n}, \frac{\partial u}{\partial n} V \cdot n\right\rangle_{\Gamma} .
$$

Concerning the third term in (3.8) we argue similarly replacing the normal derivative by the tangential derivative in the definition of $w_{k}$. Since $\nabla p_{k}$ converges to $\nabla p$ also in $H(\operatorname{curl}, \Omega)$, the tangential derivative (in $\mathbb{R}^{2}$ ) extends to a continuous linear operator in $\mathcal{L}\left(H(\operatorname{curl}, \Omega), H^{-1 / 2}(\Gamma)\right.$ ) 
[GR, Theorem 2.11]. Thus

$$
\lim _{k \rightarrow \infty} \int_{\Gamma}\left(\nabla p_{k} \cdot \tau\right)\left(\nabla u_{k} \cdot \tau\right) V \cdot n \mathrm{~d} \sigma=\left\langle\nabla p \cdot \tau, \frac{\partial u}{\partial \tau} V \cdot n\right\rangle_{\Gamma}=0,
$$

upon making use of the boundary condition $u=0$ in the last equality. Similarly one finds

$$
\lim _{k \rightarrow \infty} \int_{\Gamma} \frac{\partial p_{k}}{\partial n}\left(\nabla u_{k} \cdot \tau\right) V \cdot n \mathrm{~d} \sigma=0
$$

Summarizing we have shown the desired representation for the shape gradient of $J$ :

$$
\mathrm{d} J(\Gamma) V=-\left\langle\frac{\partial p}{\partial n}, \frac{\partial u}{\partial n} V \cdot n\right\rangle_{\Gamma}-\int_{\Gamma}\left(\frac{1}{2} p^{2}+Q p\right) \kappa V \cdot n \mathrm{~d} \sigma .
$$

In the last step of the proof, the 2-dimensionality of $\Omega$ is used. In higher dimensions the representation (3.3) remains true provided that the boundary of $\Omega$ is sufficiently regular so that $u \in H^{3}(\Omega)$ and hence $p \in H^{2}(\Omega)$, in which case it is not necessary to approximate $p$ by smoother functions $p_{k}$.

REMARK 3.1 The proof of Theorem 3.1 can be easily adapted to cover the inhomogeneous state equation

$$
\begin{aligned}
-\Delta u=f & \text { on } \Omega, \\
u=g & \text { on } \Gamma_{d}, \\
u=0 & \text { on } \Gamma .
\end{aligned}
$$

Then $u^{t}$ satisfies

$$
\left(A(t) \nabla u^{t}, \nabla \psi\right)=\left(I_{t} f_{t} \circ T_{t}, \psi\right) \quad \text { for all } \psi \in H_{0}^{1}(\Omega),
$$

$u^{t}=0$ on $\Gamma$ and $u^{t}=g$ on $\Gamma_{d}$ instead of 2.6. Assuming that $f \in H^{1}(U)$, the shape gradient of $J$ is then given by

$$
d J(\Gamma) V=-\int_{\Gamma}\left(\frac{1}{2} p^{2}+Q p\right) \kappa V \cdot n \mathrm{~d} \sigma-\left\langle\frac{\partial p}{\partial n}, \frac{\partial u}{\partial n} V \cdot n\right\rangle_{\Gamma}-\int_{\Gamma} f p V \cdot n \mathrm{~d} \sigma .
$$

The only changes in the proof arise in the discussion of $J_{31}$ which has to be augmented by the term $-\left(I_{t} f_{t} \circ T_{t}-f, p\right)$, and in the last but one step where one has to replace $\lim _{k \rightarrow \infty} \Delta u_{k}=0$ by $\lim _{k \rightarrow \infty} \Delta u_{k}=-f$.

\section{Example}

In [HKKP2] we used the analytic gradient [3.3) to solve the exterior Bernoulli problem based on the optimal shape design problem $1.1-(1.2)$. The free boundary component was represented by a piecewise quadratic Bezier curve with a fixed number of Bezier arcs. For more details on the implementation and results we refer to [HKKP2]. Here we report an interesting numerical finding which is based on the observation that the shape derivative (3.3) can equivalently be written as

$$
\mathrm{d} J(\Gamma) V=-\left\langle\frac{\partial p}{\partial n}, \frac{\partial u}{\partial n} V \cdot n\right\rangle_{\Gamma}-\frac{1}{2} \int_{\Gamma}\left(\left(\frac{\partial u}{\partial n}\right)^{2}-Q^{2}\right) \kappa V \cdot n \mathrm{~d} \sigma .
$$




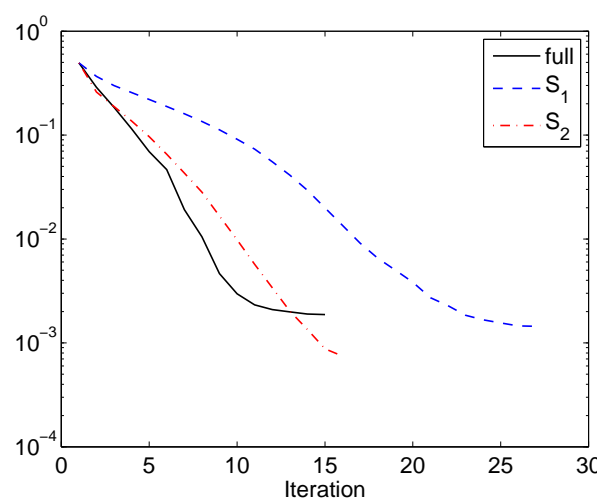

FIG. 1. Convergence history of $J$.

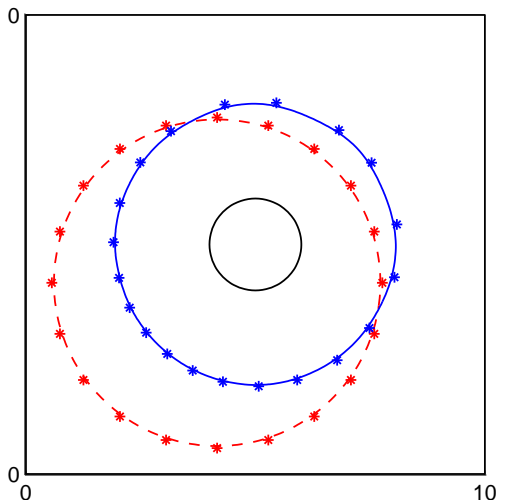

FIG. 2. Computed free boundary.

It should be noted that the second term does not depend on the adjoint variable $p$. This motivated us to test the "partial" gradients

$$
S_{1} V=-\left\langle\frac{\partial p}{\partial n}, \frac{\partial u}{\partial n} V \cdot n\right\rangle_{\Gamma}, \quad S_{2} V=-\frac{1}{2} \int_{\Gamma}\left(\left(\frac{\partial u}{\partial n}\right)^{2}-Q^{2}\right) \kappa V \cdot n \mathrm{~d} \sigma
$$

in the numerical realization of $11.1-(1.2)$. Figure 1 compares the convergence history of $J$ utilizing the full and partial gradients on a semilogarithmic scale. Figure 2 shows the computed free boundary together with the initial guess in the case of a circular fixed inner boundary with center $M(5,5)$ and radius 1 . Although it is known that for this configuration the free boundary is given by a concentric circle with radius 3 , the optimization algorithm was initialized for the sake of testing by an exocentric circle. The stars indicate the position of the control nodes for the initial and final design. In Table 1 we show the average distance $\bar{r}$ of points on the computed free boundary to $M$, the final value of $J$ and the number of iterations until termination of the algorithm (in all three cases the variance for the mean radius was of the order $10^{-4}$ ). It is noted that either gradient informationfull or partial—results in a very satisfactory approximation of the free boundary. The convergence histories of $J$ indicate that the partial gradient $S_{2}$ and the full gradient are comparable in efficiency. This behavior was not only observed in the circular case for a wide variety of initial guesses, but also in the case of an L-shaped fixed inner boundary component.

TABLE 1

\begin{tabular}{llll}
\hline & $\bar{r}$ & $J$ & $\sharp$ iter \\
\hline full & 3.04 & 0.0018 & 15 \\
$S_{1}$ & 3.05 & 0.004 & 27 \\
$S_{2}$ & 3.04 & 0.00075 & 16 \\
\hline
\end{tabular}

\section{Acknowledgements}

This research was supported in part by the Fonds zur Förderung der Wissenschaft und Forschung under SFB032 "Mathematical Optimization and Applications in Biomedical Sciences". The first and the third authors acknowledge the support of the project IAA100750802 of the grant agency of the Czech Republic and MSM0021620839. 


\section{REFERENCES}

[BG] Begis, D., \& Glowinski, R. Application de la méthode des éléments finis à l'approximation d'un problème de domaine optimal. Appl. Math. Optim. 2 (1975), 130-169. Zbl 0323.90063 MR 0443372

[DZ] Delfour, M. C., \& ZolÉsio, J.-P. Shapes and Geometries. SIAM (2001). Zbl 1002.49029 MR 1855817

[EH] EPPlER, K., \& HARBRecht, H. Efficient treatment of stationary free boundary problems. Appl. Numer. Math. 56 (2006), 1326-1339. Zbl 1104.65111 MR 2245459

[EHS] EPPler, K., HARbrecht, H., \& SChNEIDER, R. On convergence in elliptic shape optimization. SIAM J. Control Optim. 45 (2007), 61-83. Zbl pre05240370 MR 2299620

[E] Evans, L. C. Partial Differential Equations. Amer. Math. Soc., Providence (1998). Zbl 0902.35002 MR 1625845

[GR] Girault, V., \& RAVIART, P. A. Finite Element Methods for Navier-Stokes Equations. Springer, Berlin (1980). Zbl 0441.65081

[G] Grisvard, P. Elliptic Problems in Nonsmooth Domains. Pitman, Boston (1985). Zbl 0695.35060 MR 0775683

[HHM] Haslinger, J., Hoffmann, K. H., \& MÄKinen, R. Optimal control/dual approach for the numerical solution of a dam problem. Adv. Math. Sci. Appl. 2 (1993), 189-213. Zbl 0784.49005 MR 1239255

[HKKP1] Haslinger, J., Kozubek, T., Kunisch, K., \& Peichl, G. Shape optimization and fictitious domain approach for solving free boundary problems of Bernoulli type. Comput. Optim. Appl. 26 (2003), 231-251. Zbl 1077.49030 MR 2013364

[HKKP2] Haslinger, J., KozUbek, T., Kunisch, K., \& Peichl G. Fictitious domain methods in shape optimization with applications in free-boundary problems. In: Numerical Mathematics and Advanced Applications (Prague, 2003), M. Feistauer et al. (eds.), Springer (2004), 56-75. Zbl 1056.65063 MR 2120794

[HM] Haslinger, J., \& MÄKInEN, R. Introduction to Shape Optimization. SIAM Adv. Design and Control, Philadelphia (2003). Zbl 1020.74001 MR 1969772

[IKP] Ito, K., KUnisch, K., \& Peichl, G. Variational approach to shape derivatives. ESAIM Control Optim. Calc. Var. 14 (2008), 517-539. Zbl pre05309729 MR 2434064

[MS] Murat, F., \& Simon, J. Sur le contrôle par un domaine géométrique. Rapport 76015, Univ. Pierre et Marie Curie, Paris (1976).

[Ne] Nečas, J. Les Méthodes Directes en Théorie des Equations Elliptiques. Masson, Paris (1967). MR 0227584

[SZ] SokoŁowsKi, J., \& ZolÉsIo, J.-P. Introduction to Shape Optimization, Springer, Berlin (1991).

[Z] ZolÉsio, J.-P. The material derivative (or speed) method for shape optimization. Optimization of Distributed Parameter Structures, Volume II, E. J. Haug and J. Cea (eds.), Sijthoff and Noordhoff (1981), 1089-1151. Zbl 0517.73097||MR 0690991 